Open Access

\title{
Load-distributing-band cardiopulmonary resuscitation for out-of-hospital cardiac arrest increases regional cerebral oxygenation: a single-center prospective pilot study
}

Yoshihito Ogawa ${ }^{1 *}$, Tadahiko Shiozaki ${ }^{1}$, Tomoya Hirose ${ }^{1}$, Mitsuo Ohnishi ${ }^{1}$, Yasushi Nakamori ${ }^{2}$, Hiroshi Ogura ${ }^{1}$ and Takeshi Shimazu ${ }^{1}$

\begin{abstract}
Background: Despite advances in therapeutic strategies and improved guidelines, morbidity and mortality rates for out-of-hospital cardiac arrest (OHCA) remain high. Especially, neurological prognosis is one of the most important problems even though brain protection therapy for patients with OHCA has improved greatly in recent years due to the development of emergency post-cardiac arrest interventions such as mild therapeutic hypothermia, early percutaneous coronary intervention, and extracorporeal cardiopulmonary resuscitation (CPR). Recently, cerebral regional oxygen saturation $\left(\mathrm{rSO}_{2}\right)$ has received attention as a method for evaluation of cerebral oxygenation. We have reported that conventional chest compression did not improve the $\mathrm{rSO}_{2}$ of cardiac arrest patients if they did not achieve return of spontaneous circulation. It is, however, unclear whether a mechanical CPR device is helpful in improving $\mathrm{rSO}_{2}$. The purpose of this study was to evaluate the effects of load-distributingband CPR (LDB-CPR) on $\mathrm{rSO}_{2}$.

Methods: In this prospective study, LDB-CPR was begun for OHCA with the AutoPulse ${ }^{T M}$ device on patient arrival at hospital. During mechanical $\mathrm{CPR}, \mathrm{rSO}_{2}$ values were recorded continuously from the forehead of the patients. CPR for patients with OHCA was performed according to the Japan Resuscitation Council Guidelines 2010 except for using the AutoPulse ${ }^{\mathrm{TM}}$ instead of manual chest compression.

Results: From December 2012 to December 2013, 34 patients (mean age, 75.6 \pm 12.8 years) with OHCA were included in this study. Duration of time from recognition of cardiac collapse to arrival to hospital was $31.0 \pm$ 11.4 min. Compared with the $\mathrm{rSO}_{2}$ value of $38.9 \pm 0.7 \%$ prior to starting LDB-CPR, $\mathrm{rSO}_{2}$ values at 4,8 and 12 minutes increased significantly after initiation of LDB-CPR $(44.0 \pm 0.9 \%, 45.2 \pm 0.8 \%$, and $45.5 \pm 0.8 \%$, respectively, $\mathrm{p}<0.05)$.
\end{abstract}

Conclusion: $\mathrm{LDB}-\mathrm{CPR}$ significantly increased the $\mathrm{rSO}_{2}$ of cardiac arrest patients during resuscitation. Keywords: $\mathrm{rSO}_{2}$, Resuscitation, LDB-CPR

\footnotetext{
* Correspondence: yoshi.ogawa@hp-emerg.med.osaka-u.ac.jp

${ }^{1}$ Department of Traumatology and Acute Critical Medicine, Osaka University Graduate School of Medicine, 2-15 Yamadaoka, Suita, Osaka 565-0871, Japan Full list of author information is available at the end of the article
} 


\section{Background}

Sudden cardiac arrest is a leading cause of death and remains a major public health problem in the industrialized world [1]. Despite improvements in resuscitation practice including the 'chain of survival', outcomes from out-of-hospital cardiac arrest (OHCA) remain poor [2, 3]. Especially, neurological prognosis is one of the most important problems even though brain protection therapy for patients with OHCA has greatly improved in recent years due to the development of emergency post-cardiac arrest interventions such as mild therapeutic hypothermia, early percutaneous coronary intervention, and extracorporeal cardiopulmonary resuscitation (CPR) [4]. For cerebral resuscitation, it is important to maintain cerebral oxygenation during CPR. However, only a few reports have evaluated cerebral oxygenation, and there is no effective way of maintaining cerebral oxygenation with manual CPR.

Previous reports have noted that an inadequate intraoperative value of cerebral regional oxygen saturation $\left(\mathrm{rSO}_{2}\right)$ is a significant predictor of postoperative neurological complications after cardiovascular surgery $[5,6]$. Now, $\mathrm{rSO}_{2}$ can be measured noninvasively in real time by near-infrared spectroscopy (NIRS). NIRS does not require vascular pulsation and can measure $\mathrm{rSO}_{2}$ even in patients with hypotension, hypothermia, and/or circulatory arrest. $\mathrm{rSO}_{2}$ on hospital arrival is a potential novel predictor of neurological outcomes at hospital discharge in patients with OHCA.

Therefore, high-quality chest compressions are emphasized by the International Liaison Committee on Resuscitation (ILCOR) [7]. Mechanical chest compression devices have been developed to assist rescuers in providing consistent high-quality compressions. A mechanical chest compression device that uses a load-distributing band (LDB) has been shown in animal and human studies to improve hemodynamic parameters over that of manual CPR $[8,9]$. The results show that the ability to achieve return of spontaneous circulation (ROSC) with mechanical chest compression devices is significantly improved when compared with manual chest compressions. Therefore, the purpose of this study was to evaluate both the changes of $\mathrm{rSO}_{2}$ during $\mathrm{CPR}$ and the effects of $\mathrm{LDB}-\mathrm{CPR}$ on $\mathrm{rSO}_{2}$.

\section{Materials and Methods Study Population}

Osaka University Hospital, with one of the largest emergency departments in western Japan, services approximately 1 million people who reside in a $200-\mathrm{km}^{2}$ area in the suburbs, which includes a number of high-rise condominiums and some mountainous regions. The population density is approximately 10,910 persons per $\mathrm{km}^{2}$, and there are 27 fire stations with a corresponding number of emergency dispatch centers. Emergency medical services (EMS) at these fire stations are provided by municipal governments. EMS personnel are trained in all aspects of advanced life support procedures for prehospital emergency care.

We performed a single-center prospective pilot study that included patients with OHCA transferred to the emergency room of Osaka University Hospital from December 2012 to December 2013. The exclusion criteria included a patient age of $<15$ years and OHCA caused by trauma.

The study protocol complied with the guidelines for epidemiologic studies issued by the Ministry of Health, Labour and Welfare of Japan and was approved by the Ethical Committee at Osaka University (no. 12385). Every patient received the standard care available at the hospital, and no patient received any experimental intervention. In light of these safeguards, the Ethical Committee approved this study and permitted the study to be exempt from the acquisition of oral or written consent.

\section{Interventions}

All patients underwent conventional CPR with manual chest compressions delivered by EMS personnel with or without bystander CPR. After arriving at the hospital, the patients underwent conventional CPR with manual chest compressions delivered by the medical staff until LDB-CPR was started. We could not evaluate the prehospital or in-hospital quality of conventional CPR with manual chest compression. We began to measure the $\mathrm{rSO}_{2}$ of the patients with the NIRS device (TOS-OR; Fujita Medical Co., Tokyo, Japan) when they arrived in the emergency room. Patients underwent LDB-CPR by mechanical chest compression delivered by the AutoPul$\mathrm{se}^{\mathrm{TM}}$ system (ZOLL, Chelmsford, MA, USA) if they were intubated prior to hospital arrival. Patients who were not intubated at the time of hospital arrival were administered LDB-CPR from 4 minutes after intubation to exclude the effect of oxygenation by intubation. Rhythm analysis and pulse check were performed every second minute. If the rhythm was shockable, defibrillation was performed during LDB-CPR.

The AutoPuls ${ }^{\mathrm{TM}}$ system is a portable chest compression device constructed around a backboard that contains a motor that retracts a load-distributing band under microprocessor control. The band is connected to a shaft in the board. The band is tightened and loosened around the chest by the motor, which alternates the rotation of the shaft in both directions. The patient is positioned on the board, and the two broad ends of the band are placed around the patient's chest and connected to each other. The length of the band automatically adjusts to the size and the shape of the patient. The microprocessor is programmed to provide a constant 
$20 \%$ reduction in the anterior-posterior dimension of the individual patient's chest during the compression phase. The compression rate is $80 \pm 5$ min- 1 with equal periods of compression and unloading, and the device can be operated in a continuous compression mode or in a 15:2 mode. In the 15:2 mode, compressions stop for $3 \mathrm{~s}$ after every 15 have been applied to allow two ventilations to be given to the patient. In this study, all resuscitation attempts were performed in the continuous compression mode. LDB-CPR treatment was continued until ROSC or death was declared.

An NIRS device was prepared for operation before patient arrival at the hospital. Upon patient arrival, the patient's skin was thoroughly cleaned, and the sensor was carefully applied to the patient's forehead. The $\mathrm{rSO}_{2}$ values stabilized within several seconds after placement of the NIRS probes. The $\mathrm{rSO}_{2}$ values were then monitored during CPR, and the values measured at each of the points the pulse was checked were used for analysis. The normal range of cerebral $\mathrm{rSO}_{2}$ was determined to be over $60 \%$ for the following reasons. First, the oxygen saturation of arterial blood is nearly $100 \%$ and that of venous blood is $50-60 \%$. Assuming that the arterial blood occupies about one fourth of the tissue blood volume, the mean oxygen saturation in the tissue is estimated to be $60-70 \%$. When the arterial blood supply is interrupted, oxygen is consumed and the $\mathrm{rSO}_{2}$ drops to that of venous blood, around $60 \%$, and anaerobic metabolism begins. The $\mathrm{rSO}_{2}$ actually shows a biphasic decline when arterial perfusion is interrupted: $\mathrm{rSO}_{2}$ initially drops rapidly, and then the slope becomes more gradual. Second, the lower normal limit of oxygen saturation that is commonly accepted in monitoring of jugular vein blood is $55 \%$. Third, the lower limit of oxygen saturation of mixed venous blood is $60 \%$. Fourth, the range of cerebral $\mathrm{rSO}_{2}$ from 15 volunteers $(43.2 \pm$ 8.9 years, 10 men and 5 women) breathing room air was $71.2 \pm 3.9 \%$. Finally, a cardiovascular surgery study suggested that the occurrence of neurological events increased when a drop in $\mathrm{rSO}_{2}$ of below $60 \%$ was sustained for a long period in elderly people [10]. Clinical staff performed routine post-cardiac arrest interventions regardless of these measurements. All patients were treated principally according to the strategy of the Japan Resuscitation Council 2010 Guidelines.

\section{Data Collection}

After the patients were entered into the study, their $\mathrm{rSO}_{2}$ values were measured until ROSC, or LDB-CPR was stopped. The variables of age, sex, and time from the emergency call to arrival at hospital were assessed. We evaluated patient $\mathrm{rSO}_{2}$ values pre-LDB-CPR and at 4,8 , and 12 min after the start of LDB-CPR for any cause. Neurological prognosis was evaluated with the
Cerebral Performance Category (CPC) score. The presence of any serious adverse events related to LDB-CPR was recorded.

\section{Statistical Analysis}

The comparison of $\mathrm{rSO}_{2}$ values was analyzed by analysis of variance adjusted for the pre- $\mathrm{rSO}_{2}$ values as a covariate and by the Dunnett test. A $p$ value of less than 0.05 was considered to indicate statistical significance. Statistical analyses were performed with JMP Pro version 10.0.2 for Macintosh (SAS Institute Inc., Cary, NC, USA).

\section{Results}

During the study period, 48 consecutive patients fulfilled the inclusion criteria, but 8 patients with ROSC before LDB-CPR, 2 patients with a "Do Not Attempt Resuscitation" order, and 4 patients with difficulties in recording with the NIRS device were excluded. Thus, the data of the remaining 34 patients were analyzed. Table 1 shows the demographic characteristics of the 34 patients, whose mean age was $75.6 \pm 12.8$ years. Almost all of the patients suffered unwitnessed cardiac arrest at home or at a nursing home/assisting living center. The duration of time from the emergency call to patient arrival at hospital was $31.0 \pm 11.4 \mathrm{~min}$. Eight patients were intubated

Table 1 Patient characteristics

\begin{tabular}{|c|c|}
\hline Characteristic & Total $(n=34)$ \\
\hline Age, mean $\pm S D$, years & $75.6 \pm 12.8$ \\
\hline Sex, n (male/female) & $21 / 13$ \\
\hline \multicolumn{2}{|l|}{ Bystander/witness status, $\mathrm{n}$} \\
\hline No witness & 8 \\
\hline No bystander with witness & 10 \\
\hline Family members & 5 \\
\hline Others & 11 \\
\hline \multicolumn{2}{|l|}{$\begin{array}{l}\text { Initially documented rhythm at } \\
\text { scene of cardiac arrest, } \mathrm{n}\end{array}$} \\
\hline VF/pulseless VT & 4 \\
\hline PEA & 15 \\
\hline Asystole & 15 \\
\hline $\begin{array}{l}\text { Time from call to hospital arrival, } \\
\text { mean } \pm S D \text {, min }\end{array}$ & $31.0 \pm 11.4$ \\
\hline Intubation by EMS personnel, $\mathrm{n}$ & 8 \\
\hline \multicolumn{2}{|l|}{ Rhythm at $\mathrm{rSO}_{2}$ measurement, $\mathrm{n}$} \\
\hline VF/pulseless VT & 1 \\
\hline PEA & 12 \\
\hline Asystole & 21 \\
\hline Return of spontaneous circulation, $\mathrm{n}$ & 13 \\
\hline
\end{tabular}

VF: ventricular fibrillation; VT: ventricular tachycardia; PEA: pulseless electrical activity; EMS: emergency medical services; $\mathrm{rSO}_{2}$ : regional cerebral oxygen saturation. 
prior to hospital arrival. Of the 34 patients, 13 achieved ROSC (Table 1).

A typical example of the change in $\mathrm{rSO}_{2}$ values upon achieving ROSC after LDB-CPR is shown in Fig. 1. The $\mathrm{rSO}_{2}$ value increased during LDB-CPR and was maintained, and after $\mathrm{ROSC}$, the $\mathrm{rSO}_{2}$ value increased further.

Before the start of LDB-CPR, the mean $\mathrm{rSO}_{2}$ value among the patients was $38.9 \pm 0.7 \%$. Thereafter, the $\mathrm{rSO}_{2}$ value increased significantly at 4,8 , and $12 \mathrm{~min}$ after the initiation of LDB-CPR $(44.0 \pm 0.9 \%, 45.2 \pm$ $0.8 \%$, and $45.5 \pm 0.8 \%$, respectively, $\mathrm{p}<0.05$ ) (Fig. 2a). Analysis showed that the $\mathrm{rSO}_{2}$ value increased significantly in the patients who achieved ROSC in comparison with those who did not achieve ROSC (Fig. 2b, c).

Of the 13 patients with ROSC, only one patient survived to hospital discharge with a CPC score of 2. The remaining 12 patients either died or survived to hospital discharge with a CPC score of 5 . There were no complications caused by LDB-CPR in this study.

\section{Discussion}

In this study, LDB-CPR increased $\mathrm{rSO}_{2}$ values compared with manual $\mathrm{CPR}$, and $\mathrm{rSO}_{2}$ values were maintained during LDB-CPR. In our previous study, manual chest compression could not increase $\mathrm{rSO}_{2}$ without ROSC [9]. Halperin et al. reported that LDB-CPR improved hemodynamics over manual $\mathrm{CPR}$ and produced prearrest levels of cerebral flow with epinephrine in a pig model [11]. Duchateau et al. reported that a significant improvement in hemodynamics was observed during CPR when the AutoPulse ${ }^{\mathrm{TM}}$ automated chest compression device was used compared to the manual active compression-decompression $\mathrm{CPR}$ in patients with OHCA [8]. We believe that the increase in $\mathrm{rSO}_{2}$ values during LDB-CPR was effected by the hemodynamic improvement brought about by the AutoPulse ${ }^{\mathrm{TM}}$.

Kämäräinen et al. showed that the $\mathrm{rSO}_{2}$ value could not be improved by manual chest compression CPR with a feedback device [12]. In the present study, the $\mathrm{rSO}_{2}$ value pre-LDB-CPR, which reflected the value resulting from prehospital manual chest compression, was very low $(38.9 \pm 0.7 \%)$, indicating that manual chest compression could not increase the $\mathrm{rSO}_{2}$ value. Several studies have found an increase in $\mathrm{rSO}_{2}$ after ROSC in patients. The $\mathrm{rSO}_{2}$ values also increased significantly in our non-ROSC patients, which may mean that LDB$\mathrm{CPR}$ has effects that increase the $\mathrm{rSO}_{2}$ without affecting ROSC.

Hock Ong et al. reported that a resuscitation strategy using LDB-CPR in an emergency department environment was associated with improved neurologically intact survival on discharge in adults who experienced nontraumatic cardiac arrest [13]. Although it is important to maintain the $\mathrm{rSO}_{2}$ value to improve the neurological prognosis, in the present study, the neurological prognosis was very poor. As a possible reason, the time from the emergency call to hospital arrival was very long. This could be a result of the long distances and times experienced in transferring the patients living in the mountainous regions or high-rise condominiums. The changes in $\mathrm{rSO}_{2}$ values may relate to transfer distance or time. Because the $\mathrm{rSO}_{2}$ value was maintained by LDB-CPR, the neurological prognosis might have been improved if LDB-CPR had been initiated in the prehospital setting. We have just begun to study the prehospital changes in $\mathrm{rSO}_{2}$ values to help resolve this important problem.

In the CIRC trial, compared to manual CPR, LDB-CPR resulted in statistically equivalent survival and neurological

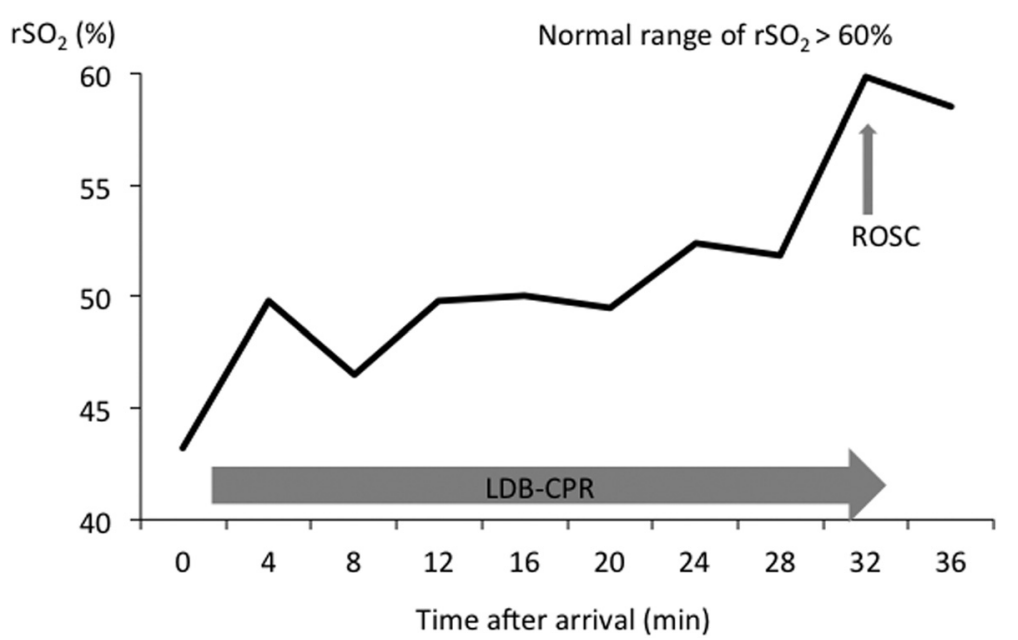

Fig. 1 A typical example of the change in $\mathrm{rSO}_{2}$ values in a patient with $\mathrm{ROSC}$ after $\mathrm{LDB}-\mathrm{CPR}$. The $\mathrm{rSO}_{2}$ value increased during LDB-CPR and was maintained. After ROSC, the $\mathrm{rSO}_{2}$ value continued to increase. $\mathrm{rSO}_{2}$ : regional cerebral oxygen saturation; ROSC: return of spontaneous circulation; LDB-CPR: load-distributing-band cardiopulmonary resuscitation. 


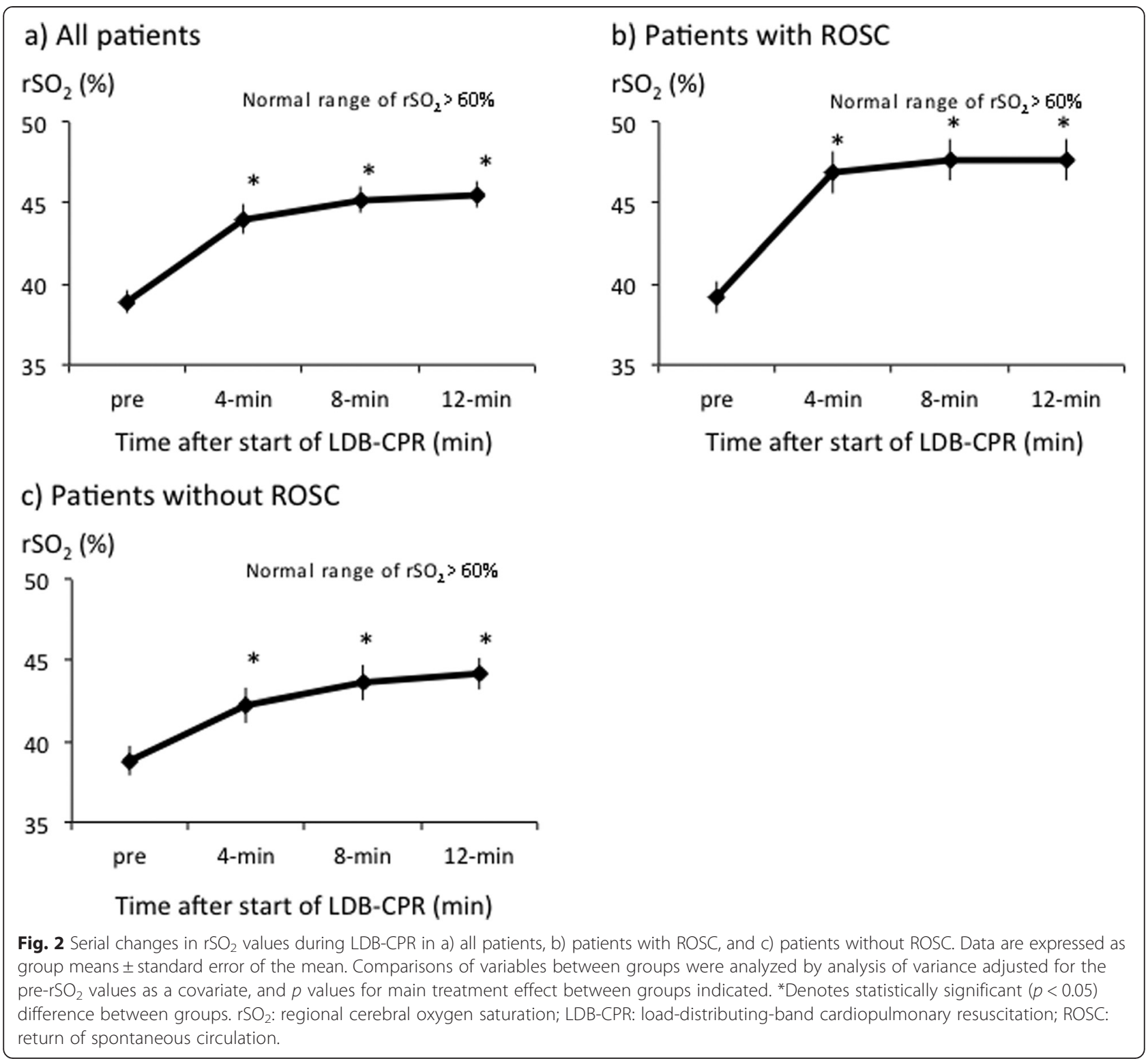

prognosis to hospital discharge after OHCA of presumed cardiac origin [14]. The different findings between the present study and the CIRC trial could be influenced by the differences in the strategy of the resuscitation guidelines. The Japan Resuscitation Council 2010 Guidelines recommend manual CPR only. In the present study, 8 patients were intubated prior to hospital arrival. Although LDBCPR in patients who were not intubated at the time of hospital arrival was begun from 4 minutes after intubation to exclude the effect of oxygenation by intubation, it is possible that the $\mathrm{rSO}_{2}$ values were increased by intubation. It is thus necessary to evaluate $\mathrm{rSO}_{2}$ values without intubation between manual CPR and LDB-CPR.

The use of mechanical chest compression devices for $\mathrm{CPR}$ remains controversial. A previous meta-analysis showed that the ability to achieve ROSC with mechanical chest compression devices is significantly improved when compared with manual chest compression [15]. In our study, LDB-CPR increased and maintained $\mathrm{rSO}_{2}$ values during resuscitation. We think that LDB-CPR may offer a significant beneficial effect on the maintenance of cerebral oxygenation during resuscitation. Moreover, we have developed a portable NIRS system and conducted a pilot study of the device [16]. This will allow us to evaluate changes in $\mathrm{rSO}_{2}$ resulting from prehospital mechanical chest compression.

We recognize several limitations in this study. First, the present study is an observational and not a randomized control study. For obvious reasons, this study could not be blinded, and it was difficult to randomize in the 
prehospital stage. Therefore, we acknowledge the necessity of comparing the changes in $\mathrm{rSO}_{2}$ values between conventional CPR with manual chest compression and LDB-CPR in the prehospital stage. Second, this is a single-center study, and third, the sample size was small. But the present study was a pilot study. It was exploratory and for this reason, the objective was 30 patients included (which has been achieved). The final limitation was that the prehospital or in-hospital quality of conventional CPR with manual chest compression could not be evaluated. There are some systems/devices that do not monitor the quality of CPR. None of these systems have been used in this study. The combination of these limitations might cause multiple unmeasured variables to account for the outcome differences observed in this study.

\section{Conclusion}

LDB-CPR significantly increased the $\mathrm{rSO}_{2}$ of patients with OHCA. LDB-CPR may have an important beneficial effect on the maintenance of cerebral oxygenation during resuscitation.

\section{Competing interests}

The authors declare that they have no competing interests.

\section{Authors' contributions}

Y.O. and T.S. designed the study. Y.O., T.H., and M.O. collected and generated the data. Y.O. wrote the first draft. Y.O., T.S., T.H., and M.O. analyzed the data. T.S., T.H., M.O., Y.N., H.O., and T.S. helped to draft the manuscript. All authors take responsibility for the data presented herein, and all are aware of, have read, and approve the manuscript being submitted to the Scandinavian Journal of Trauma, Resuscitation and Emergency Medicine. No author has any potential conflict of interest to disclose. All authors read and approved the final manuscript.

\section{Acknowledgments}

This work was supported by Grants-in-Aid for scientific research from the Ministry of Education, Culture, Sports, Science, and Technology in Japan (nos. 24390401 and 15H05007).

\section{Author details}

'Department of Traumatology and Acute Critical Medicine, Osaka University Graduate School of Medicine, 2-15 Yamadaoka, Suita, Osaka 565-0871, Japan. ${ }^{2}$ Department of Emergency and Critical Care Medicine, Kansai Medical University, 10-15 Fumizonocho, Moriguchi, Osaka 570-8507, Japan.

Received: 3 February 2015 Accepted: 7 November 2015

Published online: 14 November 2015

\section{References}

1. Travers AH, Rea TD, Bobrow BJ, Edelson DP, Berg RA, Sayre MR, et al. Part 4: CPR overview: 2010 American Heart Association Guidelines for Cardiopulmonary Resuscitation and Emergency Cardiovascular Care. Circulation. 2010;122(18 Suppl 3):S676-84.

2. Kitamura T, Iwami T, Kawamura T, Nagao K, Tanaka H, Hiraide A. Implementation Working Group for the All-Japan Utstein Registry of the Fire and Disaster Management Agency. Nationwide public-access defibrillation in Japan. N Engl J Med. 2010;362(11):994-1004.

3. Iwami T, Nichol G, Hiraide A, Hayashi Y, Nishiuchi T, Kajino K, et al. Continuous improvements in "chain of survival" increased survival after outof-hospital cardiac arrests: a large-scale population-based study. Circulation. 2009;119(5):728-34.
4. Neumar RW, Nolan JP, Adrie C, Aibiki M, Berg RA, Böttiger BW, et al. Postcardiac arrest syndrome: epidemiology, pathophysiology, treatment, and prognostication. A consensus statement from the International Liaison Committee on Resuscitation (American Heart Association, Australian and New Zealand Council on Resuscitation, European Resuscitation Council, Heart and Stroke Foundation of Canada, InterAmerican Heart Foundation, Resuscitation Council of Asia, and the Resuscitation Council of Southern Africa); the American Heart Association Emergency Cardiovascular Care Committee; the Council on Cardiovascular Surgery and Anesthesia; the Council on Cardiopulmonary, Perioperative, and Critical Care; the Council on Clinical Cardiology; and the Stroke Council. Circulation. 2008;118(23):2452-83.

5. Murkin JM, Adams SJ, Novick RJ, Quantz M, Bainbridge D, Iglesias I, et al. Monitoring brain oxygen saturation during coronary bypass surgery: a randomized, prospective study. Anesth Analg. 2007;104(1):51-8.

6. Slater JP, Guarino T, Stack J, Vinod K, Bustami RT, Brown 3rd JM, et al. Cerebral oxygen desaturation predicts cognitive decline and longer hospital stay after cardiac surgery. Ann Thorac Surg. 2009;87(1):36-44.

7. Sayre MR, Koster RW, Botha M, Cave DM, Cudnik MT, Handley AJ, et al. Adult Basic Life Support Chapter Collaborators. Part 5: Adult basic life support: 2010 International Consensus on Cardiopulmonary Resuscitation and Emergency Cardiovascular Care Science With Treatment Recommendations. Circulation. 2010;122(16 Suppl 2):S298-324.

8. Duchateau FX, Gueye P, Curac S, Tubach F, Broche C, Plaisance P, et al. Effect of the AutoPulse automated band chest compression device on hemodynamics in out-of-hospital cardiac arrest resuscitation. Intensive Care Med. 2010;36(7):1256-60.

9. Nakahori Y, Hirose T, Shiozaki T, Ogawa Y, Ohnishi M, Fujimi S, et al. Serial changes in values of cerebral regional saturation of oxygen $\left(\mathrm{rSO}_{2}\right)$ during resuscitation in patients with out-of-hospital cardiac arrest. Nihon Kyukyu Igakukai Zasshi. 2013;24(9):774-80. Abstract in English.

10. Orihashi K, Sueda T, Okada K, Imai K. Near-infrared spectroscopy for monitoring cerebral ischemia during selective cerebral perfusion. Eur J Cardiothorac Surg. 2004;26(5):907-11.

11. Halperin HR, Paradis N, Ornato JP, Zviman M, Lacorte J, Lardo A, et al. Cardiopulmonary resuscitation with a novel chest compression device in a porcine model of cardiac arrest: improved hemodynamics and mechanisms. J Am Coll Cardiol. 2004;44(11):2214-20.

12. Kämäräinen A, Sainio M, Olkkola KT, Huhtala H, Tenhunen J, Hoppu S. Quality controlled manual chest compressions and cerebral oxygenation during in-hospital cardiac arrest. Resuscitation. 2012;83(1):138-42.

13. Hock Ong ME, Fook-Chong S, Annathurai A, Ang SH, Tiah L, Yong KL, et al. Improved neurologically intact survival with the use of an automated, loaddistributing band chest compression device for cardiac arrest presenting to the emergency department. Crit Care. 2012;16(4):R144.

14. Wik L, Olsen JA, Persse D, Sterz F, Lozano Jr M, Brouwer MA, et al. Manual vs. integrated automatic load-distributing band CPR with equal survival after out of hospital cardiac arrest. The randomized CIRC trial. Resuscitation. 2014; 85(6):741-8.

15. Westfall M, Krantz S, Mullin C, Kaufman C. Mechanical versus manual chest compressions in out-of-hospital cardiac arrest: a meta-analysis. Crit Care Med. 2013;41(7):1782-913.

16. Tajima G, Shiozaki T, Izumino H, Yamano S, Hirao T, Inokuma T, et al. Portable system for monitoring of regional cerebral oxygen saturation during prehospital cardiopulmonary resuscitation: a pilot study. Acute Med Surg. 2015;2(1):48-52. 